\title{
Super refractory seizures with autoimmune encephalitis and ventilator associated pneumonia: a case report
}

\author{
A. Priya Shiva, Aswathy M. Shaji, Mohammed Fardan, K. Arun Chander Yadav*
}

Department of Clinical Pharmacology, Apollo Children's Hospital, Chennai, Tamil Nadu, India

Received: 11 January 2021

Accepted: 08 February 2021

*Correspondence:

Dr. K. Arun Chander Yadav,

Email: clinicalpharmaach_cni@apollohospitals.com

Copyright: ( $)$ the author(s), publisher and licensee Medip Academy. This is an open-access article distributed under the terms of the Creative Commons Attribution Non-Commercial License, which permits unrestricted non-commercial use, distribution, and reproduction in any medium, provided the original work is properly cited.

\begin{abstract}
Autoimmune encephalitis is an immune-mediated syndrome with sub-acute to chronic presentations, such as memory impairment, altered sensorium, behavioral abnormality, psychosis, and seizures. Treatment as such must not be procrastinated due to pending laboratory workup, as early recognition and initiation of therapy prevents long term neurological sequelae. This is a case report of an 11-year-old male who presented with neuropsychiatric symptoms, altered sensorium and high-grade fever which evolved into refractory status epilepticus and autonomic dysfunction. While his MRI had positive findings of encephalitis, he tested negative for infectious diseases and antibody panels.
\end{abstract}

Keywords: Autoimmune encephalitis, Refractory status epilepticus, Autonomic dysfunction, Altered sensorium

\section{INTRODUCTION}

Autoimmune encephalitis refers to a group of conditions that occur when the body's immune system mistakenly attacks healthy brain cells, leading to inflammation of the brain. People with autoimmune encephalitis may have various neurologic and/or psychiatric symptoms. It's a relatively new category of immune-mediated disease involving the central nervous system that demonstrates a widely variable spectrum of clinical presentations, ranging from the relatively mild or insidious onset of cognitive impairment to more complex forms of encephalopathy with refractory seizure. Treatment may involve intravenous immunosuppressive therapy.,3

Super refractory seizures are labeled to be a serious variant of status epileptics that continues or recur 24 hour or more even after the onset of anesthetic therapy, it is a lifethreatening neurological emergency. The etiology of SRSE in developing countries is dominated by central nervous system (CNS) infections and head injury compared to stroke and drug withdrawal in the developed countries. $^{2}$ The exact incidence of SRSE is unknown because of paucity of prospective studies. The current guidelines for managing SRSE are not age specific because the disease pathophysiology of prolonged seizures/SE and the anticonvulsant drug effects on neuronal receptors are the same in infants, children, and adults. ${ }^{3}$ A benzodiazepine (specifically IM/IV midazolam, IV lorazepam, or IV diazepam) is recommended as the initial therapy of choice (level A). In prehospital settings or where the three first-line benzodiazepine options are not available, rectal diazepam, intranasal midazolam, or buccal midazolam are reasonable initial alternatives (level B). For second-line therapy, the options include fosphenytoin (level U), valproic acid (level B), and levetiracetam (level $U$ ). There is no clear evidence that any one of these options is better than the others.

This case report discusses a complex case of super refractory seizures with autoimmune encephalitis and ventilator associated pneumonia.

\section{CASE REPORT}

A 11-year-old child came to emergency department with complaints of high-grade fever, altered sensorium, seizures (refractory). Patient previously on antiepileptic 
drugs like fosphenytoin, levipil, valproate and midazolam infusion for recurrent seizures prior to admission. Past medical history was unremarkable, had one episode of seizure at the age of 3 with electrolyte disturbances, followed by the same complaints after 7 years today. Family history includes death of father's brother at the age of 12 due to recurrent seizures. Antiepileptic medications were added to treat the seizure episode along with anesthetic agents like lorazepam, midazolam, fentanyl and thiopentone. Patient was kept under ventilation, feeds and drugs were introduced through nasogastric tube. Patient was diagnosed to have super refractory seizures. Suspecting viral encephalopathy patient was started on oseltamivir and acyclovir. Blood culture report for H1N1 and scrub typhus read negative and antiviral medications were stopped. Only after introduction of ketamine infusion along with lorazepam and thiopentone, seizure frequency and duration reduced from 6 episodes/day to 1 or 2 episodes/day and 5-15 sec respectively. Based on MRI and CSF report, patient was diagnosed to have autoimmune encephalopathy, which according to guidelines was treated with Injection. Methylprednisolone pulse therapy and 2 $\mathrm{g} / \mathrm{kg}$ of IV immunoglobulin therapy. On day 5 of admission, patient underwent surgery in order to introduce intracranial probe (ICP) to pinpoint the source of seizures. Therapeutic drug monitoring for phenobarbitone and phenytoin were done to ensure whether the serum concentration is within the therapeutic level. Both were within the normal range 31.54 and 12.98 respectively. Antiepileptic drugs were titrated and tapered according to the seizure activity. Due to prolonged bed-day and intubation, grade 4 pressure sore with eschar formation developed on occipital region and ventilation associated pneumonia was also witnessed. The later had been treated with ceftazidime, meropenem and fluconazole and for the former condition debrides local application was advised. He developed hyperkalemia which was managed with insulin/dextrose infusion. His urine output remained normal, Sr creatinine was 0.86 and Sr CPK was 800 . Hyperglycemia, hypernatremia was managed according to unit protocol.

Patients mean arterial pressure (MAP) was maintained at 65-70 with the help of inotropes (adrenaline, noradrenalin and vasopressin). Maintenance of normoglycemic levels and proper $\mathrm{BP}$ was necessary to prevent any further damage. On day $9^{\text {th }}$ of treatment perampanel was planned and administered which is particularly given in drug resistant seizures in order to control focal and generalized tonic-clonic seizures, the two types which were prevalent in this patient. Perampanel therapeutic approach tended to be supporting factor for the patient's treatment. Patient was not able to pass stools for past 5 days, abdominal distension was positive on day 12 , so was introduced with enema. Abdominal scans reveal subacute intestinal obstruction, for which patient was kept under NBM status with $2^{\text {nd }}$ hourly NG aspiration, Seizures subsided with normal EEG, AEDs were gradually tapered and one dose of lorazepam and phenobarbitone was given. Child developed new onset fever spikes of high grade and with no hemodynamic instability. In view of suspected, central line related infection/ventilator associated pneumonia, blood culture and BAL culture were sent and was started on piptaz. He continued to have high grade fever spikes; hence antibiotic was escalated to polymyxin B. Blood culture grew Enterobacter cloacae and BAL grew pseudomonas (insignificant growth). Due to prolonged bed days in hospital, he developed eschar formation which was treated with debrides local application which means to remove dead tissue. For those who use a wheelchair or who have been prescribed bed care, it's important to regularly reposition them to relieve stress and pressure to areas prone to skin breakdown. This may involve switching positions every 15 minutes to 1 hour. Child also had oozing from occipital area (pus) which was sent for culture and the child was started on clindamycin. Wound culture grew Acinetobacter and E. coli., hence the treatment was changed to colistin and Targocid. Fever spikes were evident to settle thereafter. Finally, the patient was discharged with mechanical ventilation along with ketamine $30 \mathrm{mcg} / \mathrm{kg} / \mathrm{min}$ and antiepileptics (levipil, fosphenytoin, valproate, phenobarbitone, perampanel.

\section{DISCUSSION}

Morbidity and mortality in autoimmune encephalitis with super refractory seizures relate directly to the delay in prompt diagnosis, proper therapeutic approach and neurosurgical intervention. Here the patient was diagnosed to have super refractory seizures along with autoimmune encephalitis. He was on antiepileptic medications which were started and later optimized according to serum levels. Ketamine infusion was added in order to get rid of recurrent seizures which were not controlled even after addition of 3 antiepileptic drugs and later Thiopentone infusion was accompanied for supportive therapy. Ketamine infusion in the management of SRSE provides a promising choice given its unique mechanism of action on the NMDA receptors, availability, fast onset of action and generally benign side effects profile. After the introduction of ketamine and Thiopentone the frequency and duration of the seizure reduced. EEG was monitored regularly; Moreover, patient's ketogenic diet and neuroprotective measures were taken care of. The addition of perampenal served as an efficient therapy here for the patient. Perampenal has been the treatment of choice for status epilepticus after failure of benzodiazepines. This favorable profile is likely explained by the glutamate and gammaaminobutyric acid (GABA) receptor composition in ongoing seizures. It highlights the use of the novel anticonvulsant perampenal in the treatment of superrefractory status epilepticus. Perampenal was effective in treatment of status epilepticus in these cases with varying etiologies which was well tolerated. ${ }^{4}$

A similar study shows that benzodiazepines like midazolam, lorazepam or diazepam are the treatment of choice and preferably should be administered as an adequate full dose rather than broken into multiple doses. Treatment options include repeating second line therapy or 
adding on anesthetic agents. Ketamine also reported to be effective in few studies of acts by inhibiting N-methyl d aspartate receptors and hence can be used as an alternative agent when common anesthetic agents fail to produce positive outcomes for status epilepticus. Ketogenic diet by its anti-inflammatory action has been successful, in few studies controlling super refractory status epilepticus, it is useful in children with GLUT-1 transporter deficiency which is a rare genetic metabolic disorder characterized by deficiency of a protein that is required for glucose (a simple sugar) to cross the blood-brain barrier. The most common symptom is seizures (epilepsy), which usually begin within the first few months of life.

Patient was diagnosed to have autoimmune encephalopathy, which was treated with injection. Methylprednisolone pulse therapy and $2 \mathrm{~g} / \mathrm{kg}$ of $\mathrm{IV}$ Immunoglobulin therapy. The aim of pulse therapy is getting quickly effective and decreasing the need for long term use of steroids. The contradiction here is that administration of high dose steroids is used to achieve the steroid sparing effect. ${ }^{5}$ Methylprednisolone pulse therapy is the intermittent administration of supraphysiological quantities of the drug to enhance its therapeutic effects while reducing the toxic effects. ${ }^{6} \mathrm{He}$ developed hyperkalemia which was managed by insulin/dextrose infusion. Intravenous insulin dextrose is often the first line therapy for acute hyperkalemia in hospitalized patients. It is typically used in conjunction with dextrose to prevent hypoglycemia.

\section{CONCLUSION}

The addition of perampanel was based on evidence-based medicine. This approach was incorporated, since the patient didn't respond to other novel antiepileptics, even with their highest end dose, blood therapeutic drug monitoring revealed antiepileptic drugs to be within normal therapeutic window. The addition of methylprednisolone pulse therapy in supraphysiological doses is evident to treat autoimmune encephalitis. Various guidelines support the aforementioned treatment approach.

\section{ACKNOWLEDGEMENTS}

Author would like to thanks department of PICU for aiding by providing all the data's necessary to incorporate in this case report.

Funding: No funding sources

Conflict of interest: None declared

Ethical approval: Not required

\section{REFERENCES}

1. Kirmani BF, Barr D, Robinson DM, Pranske Z, Fonkem E, Benge $\mathrm{J}$ et al. Management of Autoimmune encephalitis with Super Refractory Status Epilepticus. Front Neurol. 2018;259(9):1-4.

2. Dubey D, Kalita J, Misra UK. Status epilepticus: Refractory and super-refractory. Neurol India. 2017;65(7):12-7.

3. Shorvon S, Ferlisi M. A critical review of Available Therapies and a Clinical Treatment Protocol for Super Refractory Seizures. Brain. 2011;134(10):2802-18.

4. Misra UK, Kalita J, Dubey D. A Study of Super Refractory Status Epilepticus from India. Front Neurol. 2017;636(8):1-6.

5. Sinha A, Bagga A. Pulse steroid therap. Indian J Pediatr. 2008;75:1057-66.

6. Newey CR, Mullaguri M, Hantus S, Punia V, George P. Super-Refractory Status Epilepticus Treated with High Dose Perampanel: Case Series and Review of the Literature. Case Rep Cri Care. 2019;1-7.

Cite this article as: Shiva AP, Shaji AM, Fardan M, Yadav KAC. Super refractory seizures with autoimmune encephalitis and ventilator associated pneumonia-a case report. Int J Basic Clin Pharmacol 2021;10:297-9. 